Revue de droit comparé du travail et de la sécurité sociale

3 | 2018

La participation des travailleurs dans la grande entreprise privée et publique

\title{
La participation des travailleurs en Norvège et en Suède
}

Bernard Johann Mulder

\section{CpenEdition}

\section{Journals}

Édition électronique

URL : https://journals.openedition.org/rdctss/1883

DOI : $10.4000 /$ rdctss. 1883

ISSN : 2262-9815

Éditeur

Centre de droit comparé du travail et de la sécurité sociale

Édition imprimée

Date de publication : 1 novembre 2018

Pagination : 52-69

ISSN : 2117-4350

Référence électronique

Bernard Johann Mulder, "La participation des travailleurs en Norvège et en Suède », Revue de droit comparé du travail et de la sécurité sociale [En ligne], 3 | 2018, mis en ligne le 01 novembre 2021. consulté le 13 novembre 2021. URL : http://journals.openedition.org/rdctss/1883 ; DOI : https:// doi.org/10.4000/rdctss. 1883

\section{(c) $(1) \odot$}

Revue de droit comparé du travail et de la sécurité sociale est mise à disposition selon les termes de la Licence Creative Commons Attribution - Pas d'Utilisation Commerciale - Pas de Modification 4.0 International. 


\section{LA PARTICIPATION DES TRAVAILLEURS EN NORVÈGe ET EN SUÈdE}

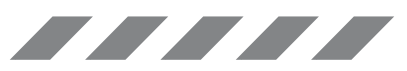

\section{ABSTRACT}

In this paper, central features in Norwegian and Swedish labour law are discussed. It stresses the significance of mutual trust and recognition between not only the labour market parties themselves, but also between these parties and the government and its authorities, without hiding the existing conflict of interests or other contests that always to some extent is challenging the Nordic labour law model. KEY WORDS : Worker Participation, Forms of Worker Influence, Nordic Model, Nordic
Labour Relations.

\section{RÉSUMÉ}

Le présent document traite des principaux aspects du droit du travail norvégien et suédois. Il souligne l'importance de la confiance et de la reconnaissance mutuelles non seulement entre les acteurs du marché du travail eux-mêmes, mais également entre ces acteurs, le gouvernement et ses représentants, sans occulter les conflits d'intérêts existant qui remettent toujours en cause, dans une certaine mesure, le modèle nordique du droit du travail.

MOTS CLÉS : Participation des travailleurs, formes d'influence des travailleurs, modèle nordique, relations de travail nordiques. 
es syndicats et les organisations patronales ont, dès la fin du XIXème siècle et tout au long du XXème, joué un rôle prédominant dans l'élaboration, le développement, le maintien et l'importance, de la législation du travail et du dialogue social dans les pays d'Europe du Nord. Dans les pays scandinaves (Danemark, Norvège et Suède), il en a été de même dans une large mesure : mouvements ouvriers luttant pour les droits des travailleurs; conflits de travail avec des grèves relativement importantes pour obtenir des droits; combat pour l'égalité des droits et le droit de vote; lock-outs menés par les employeurs avec des effets parfois dévastateurs pour les syndicats; partenaires sociaux cherchant à stabiliser le marché du travail et à réglementer leurs relations par des conventions collectives de secteur ; élaboration de compromis et de conventions collectives de base ; reconnaissance et acceptation mutuelle entre les partenaires sociaux; confiance des salariés et des employeurs dans les partenaires sociaux pour régler les conflits, chaque partenaire étant organisé sur le plan international; l'État gardant un œil attentif sur les partenaires sociaux et sur leurs activités, interférant très peu dans les relations des partenaires sociaux et de moins en moins jusqu'à la moitié du XXème siècle ${ }^{1}$. Tous ces éléments ont façonné le modèle nordique de dialogue social. Nonobstant tous ces facteurs communs, il existe des différences notoires entre chaque pays pour résoudre les conflits du travail dans la phase de développement des relations professionnelles, reflétant la manière dont les relations entre les partenaires sociaux se sont développées et les instruments mis en place pour régler les litiges liés au marché du travail sur le plan national.

1 Voir P. Wahlberg, (éd.), «Stability and Change in Nordic Labour Law», Scandinavian Studies in Law, Vol. 43, 2002. (Disponible au format numérique); N. Bruun, B. Flodgren, M. Halvorsen, H. Hydén, R. Nielsen, The Nordic Labour Relations Model. Labour Law and Trade Unions in the Nordic Countries, 1992. S. Evju, «Labour Courts and Collective Agreements - the Nordic Model», dans S. Evju, Arbeidsrett. Utvalgte artikler 2001-2010, 2010, p.77-86. (Publié également dans Europäische Zeitschrift für Arbeitsrecht 2008, 429-437.) Un aperçu des systèmes du droit du travail en Norvège et en Suède est présenté dans E. Løken, T.A. Stokke, K. Nergaard, Labour Relations in Norway, 2013. (Disponible au format numérique) ; R. Fahlbeck, B.J. Mulder, Labour and

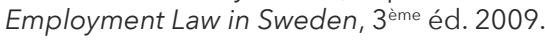


D’un point de vue géographique, la Scandinavie est une péninsule qui se compose de la partie continentale de la Norvège et de la Suède, de la partie Nord-ouest de la Finlande et d'une petite partie du Nord-ouest de la Russie. En tant que région socio-politique, dotée de traditions linguistiques et culturelles communes, la Scandinavie inclut le Danemark, la Norvège et la Suède. Les langues des trois pays sont relativement semblables si bien qu'une personne d'un pays scandinave peut en général comprendre et être comprise par une personne d'un autre pays scandinave. Le terme «Scandinavie » est généralement utilisé dans un sens socio-politique. Cependant, il se rapporte parfois de manière erronée, au sens strict, à l'ensemble des cinq pays nordiques, incluant alors la Finlande et l'Islande. Avec la Finlande et l'Islande, les régions autonomes danoises des Îles Féroé et du Groenland, et la région autonome finlandaise d'Åland, les pays scandinaves forment les pays nordiques ${ }^{2}$.

Les pays nordiques sont culturellement, historiquement, juridiquement, linguistiquement et politiquement proches les uns des autres. La Finlande a fait partie de la Suède jusqu'en 1809, année où elle est devenue autonome au sein de la Russie, avant d'obtenir son indépendance en 1917. Avant d'être incluse dans le royaume de Suède entre 1814 et 1905, avec sa propre Constitution, la Norvège a fait partie du Danemark pendant plus de quatre siècles. À l'instar de la Norvège, l'Islande est devenue une partie du Danemark en 1397 et est restée dans le giron danois jusqu'en 1944. Le Danemark est membre de I'Union européenne (UE) depuis 1973, la Finlande et la Suède depuis 1995. L'Islande et la Norvège sont membres de l'Association européenne de libre-échange (AELE) respectivement depuis 1971 et 1960 et font partie de l'Espace économique européen (EEE), comme l'Union européenne et ses États membres.

De cette évolution, se dégage un élément essentiel de la construction des relations du travail en Scandinavie qui est la confiance. Ainsi, les syndicats et les organisations patronales jouissent de la plus grande confiance et loyauté de la part de leurs membres. II existe par ailleurs un degré de confiance et de loyauté considérable entre les partenaires sociaux eux-mêmes. En outre, la confiance et la loyauté dont témoignent à leur tour le gouvernement et les autorités administratives à l'égard des partenaires sociaux ont un rôle clé dans la manière dont le marché du travail s'est développé et est désormais façonné dans les pays scandinaves.

Comme dans de nombreuses autres parties de l'Europe, le modèle nordique de dialogue social est né dans le sillage des troubles sociétaux engendrés par l'industrialisation au milieu du XIXème siècle, au moment où s'est également développé le mouvement ouvrier. À la fin du XIXème siècle et au début du XXème, la scène politique était agitée, comme ailleurs en Europe, avec des protestations en faveur de réformes sociales et du suffrage universel ${ }^{3}$.

2 Voir la page Web du Conseil nordique pour plus d'informations : http ://www.norden.org/en

3 O. Hasselbalch, «The Roots - the History of Nordic Labour law», dans P. Wahlberg, (éd.), Stability and Change in Nordic Labour Law. Scandinavian Studies in Law, Vol. 43, 2002, p. 11-35. (Disponible au format numérique). B. Hepple, (éd.), The Making of Labour Law in Europe, 1986. S. Evju, "Labour Courts and Collective Agreements - the Nordic Model», dans S. Evju, Arbeidsrett. Utvalgte artikler 2001-2010, 2010, p. 77-86. 
Bien entendu, entre les acteurs du marché du travail, les conflits d'intérêts étaient, et sont toujours, importants : le droit du travail est pour une grande part «le produit d'idéologies contradictoires $»^{4}$. Néanmoins, fortement imprégné par les conflits dès le départ, ce partenariat est basé sur une acceptation et une reconnaissance mutuelles de la coopération et sur l'acceptation par les syndicats de certaines prérogatives des employeurs. «En Suède, les employeurs n'ont jamais cherché à écraser ou à affaiblir nettement le syndicalisme. Du côté des salariés, l'acceptation pragmatique de la liberté des employeurs dans la gestion de l'entreprise et dans les évolutions technologiques est caractéristique $»^{5}$. Il est ici fait référence à la Suède, mais la situation est en principe valable pour n'importe quel autre pays nordique depuis la fin de la Seconde Guerre mondiale en 19456. II est par conséquent justifié de considérer que «la convention collective représente la méthode la plus largement utilisée à l'heure actuelle pour réglementer non seulement les salaires et les autres conditions de travail, mais aussi les relations entre les organisations du marché du travail $»^{7}$.

La réussite du modèle a été possible car l'État a adopté une attitude relativement passive et a accepté les systèmes de résolution des conflits des partenaires sociaux, sans véritablement prendre partie. Les efforts de syndicalisation et de mise en place d'une réglementation collective des salaires et des conditions de travail, comme les « efforts en faveur de la justice et de la sécurité économiques, qui ne pouvaient, du point de vue des travailleurs, être garanties selon les auteurs $\mathrm{S}$. et $\mathrm{B}$. Webb que par une « règle commune » déterminant les conditions de travail et la coopération collective pour la mise en place de cette règle commune ${ }^{8} "$ n'ont pas fait exception. Un autre facteur important pour le succès dudit modèle a été la reconnaissance par l'État, dès les premières décennies du $X X{ }^{\text {ème }}$ siècle, des méthodes de conciliation et d'arbitrage, et du statut contractuel de la convention collective, avec les mêmes effets juridiques que les autres contrats ${ }^{9}$. Cette évolution a entraîné une forte densité organisationnelle à la fois chez les salariés et chez les patrons, avec très peu de concurrence entre les opinions idéologiques et religieuses véhiculées au sein du syndicalisme ${ }^{10}$. Le taux d'organisation croissant a ouvert la voie à une forte couverture des employeurs comme des salariés par les conventions collectives, ce qui a également favorisé la progression du modèle. Il y a évidemment eu une corrélation entre la puissance grandissante des syndicats et les niveaux de reconnaissance et de

4 B. Hepple, «Introduction», dans B. Hepple, (éd.), The Making of Labour Law in Europe, 1986, p. 1-30.

5 R. Fahlbeck, et B.J. Mulder, Labour and Employmenta Law in Sweden, 3ème éd. 2009. cf. A. Adlercreutz, «The Rise and Development of the Collective Agreement», in F. Schmidt, (éd.), Scandinavian Studies in Law, Vol. 2, 1958, p. 9. (Disponible au format numérique).

6 Cf. J. Kristiansen, Den kollektive arbejdsret, 3. udgave, 2014.

7 A. Adlercreutz, «The Rise and Development of the Collective Agreement», op.cit., sp. p. 11.

8 A. Adlercreutz, The Rise and Development of the Collective Agreement, op.cit., sp. p. 47 et 49. La référence aux «Webb » est une référence à S. et B. Webb, Industrial Democracy, 1902.

9 Voir A. Adlercreutz, ibid, sp. p. 12 ; Schmidt, Law and Industrial Relations in Sweden, 1977, sp. p. 37.

10 Voir plus d'informations sur le développement de la densité syndicale depuis 1990 dans S. K. Andersen, J. E. Dølvik, C. L. Ibsen, Nordic Labour models in open markets. ETUI Report 132, 2014. (Disponible au format numérique) 
coopération obtenus. Par ailleurs, le modèle du dialogue social nordique se caractérise par une coopération et, dans une certaine mesure, par un équilibre entre capital et travail ${ }^{11}$. Au fil des années, une confiance et une loyauté mutuelles se sont installées entre les différents acteurs du marché du travail, ainsi qu'une volonté d'entretenir des relations apaisées, ce qui s'est avéré crucial pour l'essor, le développement et le maintien du modèle nordique en matière de législation du travail, et pour le rôle de premier plan que les syndicats jouent dans l'application du droit du travail.

\section{I - CARACTÉRISTIQUES CLÉS DU MODĖLE NORDIQUE DU MARCHÉ DU TRAVAIL}

Au regard de la législation du travail en Europe, on peut parler d'un modèle nordique basé sur des accords collectifs de travail comparé à un modèle continental basé sur la loi et à celui de la Common law qui s'appuie sur la jurisprudence. L'objectif de cette distinction est de souligner le fait que les pays nordiques partagent un certain nombre de caractéristiques communes par rapport aux autres pays européens. Ces caractéristiques communes sont les suivantes:

- On constate un haut niveau d'organisation à la fois chez les salariés et chez les employeurs.

- Les conventions collectives jouent un rôle important dans les systèmes du droit du travail.

- Les conventions collectives fixent normalement les salaires et les conditions de travail.

- La législation du travail joue un rôle limité par rapport à la plupart des autres pays européens.

En Suède, le taux de syndicalisation atteint environ $70 \%$, tandis qu'il est d'environ 50 \% en Norvège. Que ce soit en Norvège ou en Suède, ce taux est plus élevé dans le secteur public et chez les ouvriers. Le taux d'adhésion des employeurs à des organisations patronales est de 65 \% environ dans le secteur privé, à la fois en Norvège et en Suède. Dans le secteur public, ce taux est de $100 \%$. Le taux de couverture des conventions collectives est relativement important dans les deux pays. En Norvège, environ $70 \%$ des salariés sont couverts par des conventions collectives et, en Suède, ce taux atteint $90 \%$, en raison du fort niveau d'organisation chez les employeurs suédois ${ }^{12}$.

11 N. Bruun, B. Flodgren, M. Halvorsen, H. Hydén, et R. Nielsen, The Nordic Labour Relations Model. Labour Law and Trade Unions in the Nordic Countries, 1992.

12 Voir A. Kjellberg, Kollektivavtalens täckningsgrad samt organisationsgraden hos arbetsgivarförbund och fackförbund, 2018. (Disponible au format numérique). Ch. Nergaard, Organisasjonsgrader, tariffavtaledekning og arbeidskonfliker, 2014. (Disponible au format numérique) 
Ce qui est frappant dans le système nordique des conventions collectives, c'est que ces accords engagent à la fois les parties et leurs membres. L'obligation de paix sociale, liée aux conventions collectives, s'applique aux deux parties aux accords, à savoir aux syndicats d'un côté et aux organisations patronales ou à un employeur de l'autre. L'obligation de paix sociale s'applique également aux membres des organisations qui concluent les accords, à savoir les organisations patronales ou les syndicats locaux et les salariés adhérents de la partie contractante ${ }^{13}$. Les employeurs liés par ces contrats sont tenus de respecter les salaires et les conditions de travail des conventionnels pour tous les salariés du secteur professionnel considéré. À l'inverse, les pays nordiques ont recours à différents mécanismes pour élargir les accords professionnels afin de couvrir les employeurs non membres de l'organisation patronale signataire.

En Suède, il incombe aux syndicats de conclure des accords (« hängavtal », accords d'adhésion) avec les employeurs non membres de l'organisation patronale (employeurs non organisés $)^{14}$. Cela offre un espace de résolution des conflits sociaux, y compris des grèves de solidarité. En Norvège, il existe un système d'accords généralement applicables ${ }^{15}$. Les syndicats sont autorisés à avoir recours au conflit social comme en Suède, mais un mécanisme a été mis en place pour rendre certaines parties des conventions collectives généralement applicables afin de s'assurer que les conditions des salariés étrangers soient équivalentes à celles des salariés norvégiens et d'empêcher tout dumping social sur le marché du travail norvégien.

II n'existe pas de salaire minimum réglementaire dans les deux pays, hormis le salaire fixé par les accords généralement applicables en Norvège. En Suède, on considère que c'est aux partenaires sociaux de le déterminer par conventions collectives et par contrats de travail individuels. La libre circulation des travailleurs et des services nourrit des discussions sur la concurrence déloyale créé par des salaires tirés vers le bas et par le dumping social. II convient aussi d'interpréter ces inquiétudes à la lumière de la décision de la Cour de justice de l'Union européenne rendue à l'occasion de l'affaire Laval.

Il existe également des différences quant au niveau de protection dans divers domaines, en ce qui concerne le droit de licenciement des salariés, par exemple. En la matière, les employeurs sont plus libres de rompre le contrat de travail au Danemark et en Islande qu'en Norvège ou en Suède notamment. Non seulement le niveau d'exigence en termes de validité du motif du licenciement est plus élevé, mais un salarié licencié peut aussi rester en poste s'il remet en cause l'objectivité de la rupture de contrat devant les tribunaux.

13 Cf. Loi sur les conflits du travail de 2012 Section 6 et 8 (Norvège); Loi de codétermination de 1976 Section 26, 27 et 41 (Suède).

14 A. Adlercreutz, B.J. Mulder, Svensk arbetsrätt, 14 ${ }^{\mathrm{èm}}$ ed. 2013, sp. p. 114 et 118.

15 Cf. S. Evju, «Almengjøring på norsk», Arbeidsrett og arbeidsliv, 2009, p. 1-39. (Disponible au format numérique.) Également dans S. Evju, Arbeidsrett. Utvalgte artikler 2001-2010, 2010, 299-324. 
Le modèle du marché de travail dans les pays nordiques présente un autre point commun : les partenaires sociaux exercent une influence considérable sur le contenu de la législation du travail. Cela repose sur une longue tradition consistant à s'assurer que la législation du travail est conçue dans le cadre d'un effort collaboratif du gouvernement et des partenaires sociaux. L'intégration des partenaires sociaux prend plusieurs formes : ils peuvent ainsi participer à des comités d'experts et à des travaux d'enquête ou dialoguer directement avec les ministres concernés.

Les organisations professionnelles jouent un rôle clé dans le règlement des conflits du travail dans tous les pays nordiques. Les parties signataires des conventions collectives sont responsables de leur application et sont en mesure de sanctionner les parties en cas de violation des accords affectant les salariés.

Les litiges relatifs aux droits basés sur les lois et les accords sont réglés devant le juge. En Suède, le tribunal du travail est compétent pour l'ensemble du droit du travail. Dans les autres pays nordiques, la compétence couvre les litiges qui concernent ou résultent d'une convention collective. Les autres litiges doivent être portés devant les tribunaux de droit commun.

\section{II - LA TRADUCTION JURIDIQUE DE LA VERSION NORDIQUE DU LAISSEZ-FAIRE}

Le système juridique nordique a permis le développement d'une autre caractéristique de la législation du travail dans les pays nordiques : I'autorégulation, avec une faible intervention de l'État dans les relations du travail, encore plus faible en Suède qu'en Norvège ${ }^{16}$. L'autorégulation, basée sur la confiance et la loyauté entre les partenaires sociaux, est un trait marquant en Norvège et en Suède ${ }^{17}$. Ce modèle repose en grande partie sur des conventions collectives entre les partenaires sociaux, qui réglementent l'ensemble des relations professionnelles, mais aussi les conditions des contrats de travail individuels.

16 Voir N. Bruun, B. Flodgren, M. Halvorsen, H. Hydén, et R. Nielsen, The Nordic Labour Relations Model. Labour Law and Trade Unions in the Nordic Countries, 1992. A. Kjellberg, «Self-regulation versus State Regulation in Swedish Industrial Relations», dans M. Rönnmar, V. J. Julén Votinius, (éd.), Festskrift till Ann Numhauser-Henning, 2017, 357-383. (Disponible au format numérique)

17 T. Bergh, «Hovedavtalen og den norske arbeidslivsmodellen», dans T. Bergh, Avtalt spill, 2010, p. 13-35. A. Kjellberg, "Self-regulation versus State Regulation in Swedish Industrial Relations», dans M. Rönnmar,V. J. Julén Votinius, (éd.), Festskrift till A. Numhauser-Henning, 2017, 357-383. (Disponible au format numérique) 
La version nordique du «laissez-faire collectif " $^{18} n^{\prime}$ est pas seulement une conséquence des aspects économique et politique du libéralisme ${ }^{19}$ dont les impacts négatifs ont rapidement été observés: dans le traité de Versailles de 1919, il était indiqué que «le travail ne doit pas être considéré simplement comme une marchandise ou un article de commerce » et la Déclaration de Philadelphie de 1944 rappelle que « le travail n'est pas une marchandise $»^{20}$. Le fait que le travail ne soit pas une marchandise n'est plus actuellement un postulat incontesté : le modèle de "flexisécurité », le travail intérimaire, le contrat zéro heure, les (nouvelles) formes de travail dans l'économie partagée et la mobilité du travail soulignent ce phénomène. En outre, la réglementation des salaires et des conditions de travail par les conventions collectives a permis aux employeurs d'obtenir la paix sociale en échange ${ }^{21}$. L'autorégulation peut également être perçue comme une conséquence du scepticisme du mouvement des travailleurs à l'égard de la société dite " bourgeoise", qui englobe à la fois l'État comme législateur, juge (pouvoir judiciaire), et employeur. Cependant, l'intégration de représentants des salariés et des patrons dans des tribunaux du travail spéciaux a contribué à faire accepter le système par ses opposants ${ }^{22}$.

Les partenaires sociaux ont une autorité considérable. Les organisations gèrent les droits de chaque membre, autrement dit le salarié ou l'employeur, dans une large mesure à travers les conventions collectives. De plus, les conventions collectives, en raison d'un taux de syndicalisation élevé chez les travailleurs comme chez les patrons ${ }^{23}$, ont une portée normative pour ce qui est des conditions d'emploi. En Norvège et en Suède, l'employeur est obligé, vis-à-vis du syndicat avec lequel il est lié par une convention collective, d'appliquer les conditions de la convention collective à tous les salariés couverts par celle-ci : on dit que la convention collective a un effet normatif.

18 O. Hasselbalch, «The Roots - the History of Nordic Labour law», dans P. Wahlberg, (éd.), Stability and Change in Nordic Labour Law, Scandinavian Studies in Law, Vol. 43, 2002, p. 11-35. (Disponible au format numérique). R. Fahlbeck, "Industrial Relations and Collective Labour Law», dans P. Wahlberg, (éd.), Stability and Change in Nordic Labour Law. Scandinavian Studies in Law, Vol. 43, 2002, p. 89-133. (Disponible au format numérique). A. Jacobs, "Collective Self-Regulation», dans B. Hepple, (éd.), The Making of Labour Law in Europe, 1986, p. 193-241. O. Kahn-Freund, «Labour Law», dans M. Ginsberg, (éd.), Law and Opinion in England in the 20 ${ }^{\text {th }}$ Century, 1959, 215-263. Th. Ramm, «Laissez-faire and State Protection of Workers», dans B. Hepple, (éd.), The Making of Labour Law in Europe, 1986, p. 73-113.

19 Cf. A. Bogg, «Brexit, Labour Law and the Twilight of Liberalism», dans K. Ahlberg, N. Bruun, (éd.), The New Foundations of Labour Law, p. 37-57.

20 J.M. Servais, "International Labour Law », dans International Encyclopedia for Labour Law and Industrial Relations, mise à jour numérique 2017, sp. p. 24 f. S. Evju, «Labour Is Not a Commodity : Reapprising the Origins of the Maxim», European Labour Law Journal, 2013, p. 222-229. (Voir une version antérieure «Labour is not a Commodi»).

21 Cf. A. Adlercreutz, «The Rise and Development of the Collective Agreement», in F. Schmidt, (éd.), Scandinavian Studies in Law, Vol. 2, 1958, p. 9. (Disponible au format numérique)

22 Voir A. Adlercreutz, «The Rise and Development of the Collective Agreement», op.cit. ; A. Jacobs, "Collective Self-Regulation», dans B. Hepple, (éd.), The Making of Labour Law in Europe, 1986, p. 193-241.

23 A. Kjellberg, Kollektivavtalens täckningsgrad samt organisationsgraden hos arbetsgivarförbund och fackförbund, 2018. (Disponible au format numérique); Ch. Nergaard, Organisasjonsgrader, tariffavtaledekning og arbeidskonfliker, 2014. (Disponible au format numérique). S. K. Andersen, J. E. Dølvik, C. L. Ibsen, Nordic Labour models in open markets. ETUI Report 132, 2014. (Disponible au format numérique) 
Dans le modèle norvégien et suédois, ce système basé sur les conventions collectives est essentiel pour la réglementation des salaires et des autres conditions de travail. Dans une certaine mesure, le système est toutefois remis en cause par la nécessité d'appliquer la législation de l'Union européenne. En 1985, la Cour de justice de l'Union européenne (CJUE) a accepté que les conventions collectives soient une source de mise en œuvre d'une directive, sous réserve que l'État membre garantisse efficacement les protections ou les droits établis par la directive à tous les salariés relevant de son champ d'application, (cf. art. 153.3 TFUE) ${ }^{24}$. Ainsi, s'est développée au Danemark une pratique qui consiste à laisser les partenaires sociaux mettre en œuvre la directive par un accord collectif avant de promulguer une loi pour les salariés non couverts par l'accord ${ }^{25}$. Aucune méthode spécifique n'est développée en Suède, étant donné que le législateur met régulièrement en œuvre si nécessaire par la loi les directives de l'UE en matière de droit du travail ${ }^{26}$. On a observé les conséquences d'une mise en œuvre inappropriée lorsque le législateur suédois n'a pu prévoir la manière dont la CJUE allait interpréter la directive 96/71/CE sur le détachement de travailleurs, interprétée dans l'arrêt très critiqué rendu par la Cour dans l'affaire Laval27. En l'espèce, le tribunal du travail de Suède a d'abord pris la décision provisoire d'approuver les grèves menées par les syndicats. Par la suite, le tribunal du travail a fait référence à une décision préliminaire de la CJUE qui concluait que les grèves étaient illégales. Après cela, faisant référence à l'arrêt Laval de la CJUE, le tribunal du travail est parvenu à la conclusion que les grèves n'étaient pas légales et que les syndicats devaient par conséquent payer des dommages et intérêts ${ }^{28}$.

24 Voir Commission contre Italie, 91/81, EU :C :1982 :212.; Commission contre Danemark, 143/83, EU :C :1984 :34; Commission contre Belgique, 215/83, EU :C :1985:14. Andersen, C-306/07 Andersen, EU :C :2008 :74. Cf. R. Nielsen, EU Labour Law, 2013, p. 154-159; Nyström, EU och arbetsrätten, 66-77. B. Nyström, EU och arbetsrätten, 5 uppl. 2017, sp. p. 66-77.

25 Voir J. Kristiansen, Den kollektive arbejdsret, 3. udgave, 2014, p. 54 f.

26 Voir B. Nyström, EU och arbetsrätten, 5 uppl. 2017, sp. p. 66-77.

27 Laval un Partneri Ltd., C-341/05, EU :C :2007 :809. Voir également Viking, C-438/05, EU :C :2007 :772; Rüffert, C-346/06, EU :C :2008:189; Commission contre Luxembourg, C-319/06, EU :C :2008:350; C-271/08 Commission contre Allemagne, EU :C :2010:426. Pour plus d'informations sur la loi de mise en œuvre suédoise Lex Laval, voir le travail préparatoire de la législation modifiée dans le projet de loi du gouvernement, prop. 2016/17:107. Voir également pour l'impact de ces affaires sur la loi norvégienne S. Evju, "Norway », dans M. Freedland, J. Prassl, (éd.), Viking, Laval and Beyond, 2015, p. 211-228; sur la loi suédoise : O. Edström, «Laval - The aftermath?», dans H. Persson, Annina H. et Ryberg-Welander, Lotti, Festskrift, C. Calleman - i rättens utkanter, 91-100. Vérifier les noms et prénoms des auteurs Pour plus d'informations et une présentation générale, voir S. Evju, (éd.), Regulating Transnational Labour in Europe. The quandaries of multilevel governance, 2014. (Disponible au format numérique); M. Freedland, et J. Prassl, (éd.), Viking, Laval and Beyond, 2015.

28 Voir (Rapports du tribunal du travail) AD 2004 nr 111, AD 2005 nr 49, Laval un Partneri Ltd, C-341/05 et AD $2009 \mathrm{nr}$ 89, respectivement. Pour plus d'informations, voir O. Edström, «Laval The aftermath?», dans Persson, Annina H. et Ryberg-Welander, Lotti, Festskrift till Catharina Calleman - i rättens utkanter, 91-100. 
On peut considérer que la solution norvégienne se situe, de manière générale, entre la solution danoise et la solution suédoise. La Norvège n'est pas membre de l'Union européenne, mais est néanmoins signataire de l'Accord sur l'Espace économique européen (EEE) et est liée par cet accord au marché unique de l'UE. La législation du travail de l'UE est donc applicable en Norvège. Toutefois, l'Accord sur l'EEE n'est pas, comme le TUE et le TFUE, un ensemble de lois supranationales, mais plutôt du droit international public. Du fait du système juridique dualiste, comme au Danemark et en Suède, il est nécessaire d'intégrer ou de transposer le droit international dans le système juridique norvégien pour lui donner effet. Ceci étant, la loi norvégienne est dans une large mesure conforme à la loi de l'UE. La solution réside ici, en plus de la législation, dans l'applicabilité erga omnes des conventions collectives. Toutefois, la méthode d'extension des conventions collectives en Norvège diffère de la méthode finlandaise ou allemande, réduisant leur portée à une zone géographique ou à un secteur spécifique ${ }^{29}$.

Il ne faut pas confondre autorégulation et déréglementation. La réglementation du droit du travail, qu'il s'agisse d'autorégulation ou de régulation par l'État, joue un rôle important bien au-delà des relations de travail : «En matière de droit du travail, les normes sont sans aucun doute une condition importante pour une économie de marché qui fonctionne et surtout pour un développement durable $»^{30}$. Le modèle nordique du dialogue social assure une grande stabilité sur le marché du travail et des relations professionnelles. En raison non seulement de la tolérance et de la reconnaissance réciproques, mais aussi de la confiance mutuelle et du climat de coopération, l'État n'a nul besoin d'administrer les relations du travail quotidiennes avec un corps d'inspection du travail de l'Etat. Au contraire, l'intervention de l'État dans les relations professionnelles et sociales est limitée au minimum dans les pays nordiques, laissant aux partenaires sociaux la responsabilité de la réglementation et de l'application du droit du travail.

Si l'autorégulation est une caractéristique distinctive des marchés du travail dans les pays scandinaves, les partenaires sociaux coopèrent néanmoins avec l'État. Ainsi, les partenaires sociaux se retrouvent dans des institutions gouvernementales en lien avec le marché du travail et notamment dans les tribunaux du travail. Cependant, en Suède, l'élément néocorporatiste est plus ou moins réduit aux tribunaux du travail, les employeurs s'étant retirés des organismes publics où ils siégeaient dans les années 1990. Le dialogue social en Suède se fait donc de manière bipartite. En Norvège, l'État joue un rôle plus actif dans le dialogue social et on peut objectivement définir le dialogue social en Norvège comme reposant sur une concertation tripartite.

29 Cf. S. Evju, «Almengjøring på norsk», Arbeidsrett og arbeidsliv, 2009, p. 1-39. (Disponible au format numérique) Également dans S. Evju, Arbeidsrett. Utvalgte artikler 2001-2010, 2010, p. 299-324.

30 M. Weiss, "Globalisieriung des Arbeitsrechts und der Arbeitsbeziehungen» dans M. Jens, M. Schubert (éd.), Anforderungen an ein modernes kollektives Arbeitsrecht, Festschrift für Otto Ernst Kempen, 2013, p. 384-400, sp.p.385. 
L'évolution du système d'autorégulation des relations du travail dans un climat coopératif de longue date dans les pays nordiques a éliminé toute nécessité de mécanismes de contrôle externes aux partenaires sociaux pour l'application du droit du travail. Relativement puissants, les syndicats au niveau d'un pays, d'un secteur, d'une entreprise ou d'un site en sont arrivés à un point où ils peuvent faire eux-mêmes office d'instances de contrôle. Dans les pays nordiques, les lois et les conventions collectives étaient, et sont toujours dans une large mesure, appliquées via les systèmes de relations du travail prévalant entre les partenaires sociaux. Le passage du collectivisme à l'individualisme comme méthode d'application du droit du travail n'a pas véritablement triomphé dans les pays nordiques, comme cela a été le cas sur le continent européen et dans les îles britanniques. De manière générale, il n'y a pas eu de déclin du collectivisme. Cependant, la décentralisation des droits des salariés des syndicats vers l'individu, associée à l'activisme judiciaire, à la fois au niveau national et au niveau de l'UE, ont contribué à une juridicisation des droits du travail dans les pays nordiques ${ }^{31}$. Par ailleurs, les droits humains fondamentaux, sur lesquels on a beaucoup insisté depuis le début du nouveau millénaire, conduisent à un certain individualisme. C'est le salarié lui-même, et non son syndicat, qui doit faire valoir ses droits, lesquels doivent être appliqués et mis en œuvre par et à travers la loi et les tribunaux nationaux.

Ce que nous venons de décrire procède toutefois d'une vision générale. En Suède, le modèle se distingue par la coopération entre les partenaires du marché du travail, avec une intervention minimale du gouvernement depuis le début de la régulation des relations du travail. La naissance du modèle suédois en 1938 (également connu sous le nom de «Saltsjöbaden ») a initié le bipartisme, ce qui a permis aux partenaires du marché du travail de réguler le dialogue social. Cette non-intervention de l'État a duré jusque dans les années 1970, qui ont connu ce que l'on peut appeler une "explosion législative». Plusieurs lois ont alors été adoptées pour réglementer la protection de l'emploi, la codétermination, l'environnement de travail et l'interdiction de toute discrimination basée sur le sexe. Cependant, la plupart de ces dispositions légales ont été rendues facultatives. C'est ainsi que les partenaires sociaux liés les uns aux autres par la convention collective ont pu continuer à réguler leurs interactions, ce qu'ils font encore largement. La non-intervention du gouvernement est encore plus visible au Danemark ${ }^{32}$. Par opposition, l'État est depuis le début très fortement présent dans les relations du travail en Norvège et joue un rôle actif dans les négociations salariales à l'échelle nationale ${ }^{33}$ et dans l'arbitrage obligatoire du règlement des conflits ${ }^{34}$. Un organisme gouvernemental spécial appelé "Rikslønnsnemnda » (Conseil national sur les salaires) est en charge d'interférer dans un conflit de travail et de décider de son terme, si le conflit a des conséquences pour la vie ou la santé, ou a d'autres effets néfastes sur la société ${ }^{35}$.

31 Voir N. Bruun, «The Future of Nordic Labour Law», dans P.Wahlberg, Peter (éd.), «Stability and Change in Nordic Labour Law», Scandinavian Studies in Law, Vol. 43, 2002, p. 375-385. (Disponible au format numérique)

32 Voir J. Kristiansen, The growing conflict between European uniformity and national flexibility. The case of Danish flexicurity and European harmonisation of working conditions, 2015.

33 Voir NOU 2016: 15

34 K. Alsos, «Tvungen lønnsnemnd - finnes det alternativer?», Arbeidsrett og arbeidsliv, 2010, p. 123154. (Disponible au format numérique)

35 Cet arrangement n'est pas considéré comme étant en conflit avec la loi internationale, voir Fédération des syndicats de travailleurs offshore et autres contre Norvège, jugement 27 juin 2002, numéro d'application 38190/97. 


\section{III - LA DÉMOCRATIE AU TRAVAIL : LES FORMES D’INFLUENCE DES TRAVAILLEURS}

En Norvège et en Suède, l'influence des travailleurs se manifeste à différents niveaux et sous diverses formes pour diverses questions, du niveau central au national, en passant par le niveau sectoriel et le niveau local (site d'une entreprise privée ou d'une agence publique), s'exerçant par l'intermédiaire de représentants des salariés directement désignés par les salariés ou les syndicats, ou par les salariés eux-mêmes. Bien entendu, il fauttraiter les diverses questions aux différents niveaux et considérer les diverses formes d'influence davantage comme des méthodes complémentaires de l'influence des travailleurs que comme des alternatives. De plus, les différentes formes impliquent une influence sur différents sujets, comme la négociation salariale centrale, les questions managériales quotidiennes et à long terme, les questions relatives à l'environnement de travail, aux salaires, etc. Néanmoins, il est important de garder à l'esprit qu'elles visent diverses formes d'influence, qu'elles ont un cadre juridique différent et qu'elles possèdent un contexte historique et juridique différent. Comme indiqué précédemment, il n'existe pas de différence majeure en ce qui concerne l'influence des travailleurs entre le secteur privé et le secteur public. Cela se rapporte également au droit à l'action collective, droit fondamental, si l'influence des travailleurs se veut être plus qu'une "supplication collective» (kollektives Betteln) ${ }^{36}$.

La notion de démocratie sur le lieu de travail implique le droit pour les salariés et les syndicats d'exercer une influence sur les décisions de l'employeur. La notion suppose donc une délimitation de certaines questions devant être discutées à un niveau spécifique. En principe, la participation des travailleurs au règlement des conflits et aux négociations collectives n'est pas incluse ici. Au lieu de cela, l'influence des travailleurs vise l'implication et la codétermination pour compenser le processus décisionnel de l'employeur. Le fait qu'elle contrebalance les pouvoirs des employeurs est clair et incontesté. II ne s'agit pas de transférer les pouvoirs des employeurs aux salariés ou aux syndicats. L'expérience suédoise des fonds de salariés mis en place dans les années 1980, qui entendait assurer la démocratie économique en donnant aux salariés de l'influence via la propriété de leur entreprise, a été annulée quelques années plus tard. Il convient plutôt de discuter des pouvoirs de direction pour une négociation entre employeur et salariés/syndicats.

L'acceptation mutuelle et la négociation concertée entre employeur et salariés et leurs représentants est un trait commun à tous les pays nordiques. Les syndicats jouent un rôle crucial dans le système d'implication des travailleurs. En Norvège et en Suède, on reconnaît deux niveaux d'influence : influence au niveau du site et influence dans le conseil de surveillance. II existe à cet égard une différence entre la Norvège et la Suède. En Norvège, un organisme de codétermination spécifique appelé "bedriftsforsamling " (assemblée d'entreprise) est mis en place via les conventions collectives. Composée de salariés et d'actionnaires, cette assemblée est un organisme qui se situe entre l'assemblée générale et le conseil de surveillance, mais ne remplace pas les représentants des salariés dans les

36 BAG, 1 AZR 611/11, 54 ; BAG, 1 AZR 179/11, 117 ; cf. S. Estreicher, "Collective bargaining or 'collective begging'? Reflections on Antistrikebreaker Legislation», Michigan Law Review, 1995, p. $577-608$, sp. p. 599. 
conseils. En Suède, l'implication des travailleurs et la codétermination ont été établies par la loi sur la codetermination-Codetermination Act - de 1976. Selon la loi, I'implication des travailleurs et la codétermination s'appuient sur une négociation régulière de l'influence des travailleurs. Il s'agit de la solution suédoise sur l'implication des travailleurs. Que ce soit en Norvège ou en Suède, la participation des travailleurs aux conseils de surveillance est garantie par une législation adoptée au début des années 1970; mais dans aucun des deux pays cette forme d'implication n'est la plus importante du point de vue de l'influence des travailleurs.

Dans une certaine mesure, les formes d'influence des travailleurs reflètent les systèmes de gouvernance d'entreprise des pays. En principe, la structure des conseils d'administration dans le droit des sociétés des pays scandinaves se situe entre le système à un niveau, qui prédomine dans les pays dont la loi est d'inspiration anglo-américaine, et le système à deux niveaux, caractéristique de l'Allemagne et des autres pays continentaux en Europe ${ }^{37}$. Les modèles de gouvernance d'entreprise des pays scandinaves trouvent leur origine dans les premières lois relatives aux sociétés à responsabilité limitée adoptées à la fin du XIXème et au début du XXème siècle. La structure organisationnelle dans les lois norvégienne et suédoise sur les sociétés à responsabilité limitée demeure relativement semblable nonobstant des différences importantes sur d'autres aspects de la société.

Le modèle de gouvernance d'entreprise scandinave est un système strictement hiérarchique, divisé entre les actionnaires d'un côté, la direction et l'administration de l'entreprise de l'autre. Les actionnaires constituent l'autorité suprême de l'entreprise à travers l'assemblée générale. L'administration de l'entreprise et la gestion quotidienne des affaires incombent respectivement au conseil d'administration et au directeur, le Présidentdirecteur général (PDG ; « daglig leder » ou « administrerende direktør » en norvégien et " verkställande direktör », « vd » en suédois). Les actionnaires élisent en assemblée générale le conseil d'administration qui nomme ensuite le PDG, qui sera soumis à son contrôle. Le conseil d'administration a la responsabilité de l'administration et de la gestion. Le PDG gère les affaires quotidiennes, mais le conseil d'administration prendra les décisions à la place du PDG si le sujet est inhabituel ou particulièrement important. Le PDG est supervisé par le conseil d'administration.

Au sein de l'UE, le système de gouvernance d'entreprise a fait récemment l'objet d'une harmonisation par la directive générale sur les sociétés ${ }^{38}$. Cependant, le législateur de I'UE n'est pas parvenu à harmoniser les différents systèmes de gouvernance d'entreprise en vigueur dans les différents les États membres de l'UE. Cela signifie, par exemple, que l'entreprise est libre de choisir entre les réglementations des États membres sur cet aspect ${ }^{39}$.

37 Cf. D. Block, A.M. Gerstner, «One-Tier vs. Two-Tier Board Structure. A Comparison Between the United States and Germany, Comparative Corporate Governance and Financial Regulation», Paper 1, 2016. (Disponible au format numérique) P. K.Andersen, et al., «European Model Company Act, Nordic and European Company Law» Working paper 16-26, Première éd. 2017. (Disponible au format numérique.)

38 Directive (UE) 2017/1132 relative à certains aspects du droit des sociétés.

39 Cf. Centros, C-212/97, EU :C :1999 :126; Inspire Art, C-167/01, EU :C :2003:512 ; Sevic, C-411/03, EU :C :2005:762. 
Les syndicats disposent d'un réel pouvoir central intégré dans les relations du travail en Norvège et en Suède combiné à une décentralisation effective du domaine de l'influence des travailleurs. L'influence des travailleurs porte sur : l'information et la consultation, la codétermination, la négociation collective, la représentation au niveau du conseil, le comité d'entreprise et les activités relatives à l'environnement de travail. Ces domaines sont l'objet de réglementations distinctes.

En Norvège comme en Suède, il existe des règles sur la participation des travailleurs. En Norvège, elles ont été formulées dans les accords de base tandis qu'en Suède, elles sont devenues une partie de la législation avec la loi de codétermination de 1976. Cependant, étant donné qu'elle fait partie de l'Espace économique européen (EEE), la Norvège doit appliquer la législation de I'UE et le fait la plupart du temps grâce à des règles légales. Bien qu'une législation complète ait régi la participation des travailleurs en Suède dans les années 1970, notamment avec la loi de codétermination de 1976, l'intention du législateur était et, est toujours, de voir les partenaires sociaux convenir de l'application de la participation des travailleurs par convention collective. Par conséquent, la législation devient dans une large mesure facultative.

Dans les deux pays, le système du dialogue social opère à trois niveaux : national, sectoriel et local. Pour ce qui est de la participation des travailleurs dans l'entreprise, le niveau local revêt une importance primordiale. Concernant la représentation des salariés, il convient de souligner qu'il existe une différence essentielle entre la Norvège et la Suède. En Suède, le syndicat qui a une convention collective sur le lieu de travail jouit d'une situation particulièrement privilégiée. Les règles sur la participation des travailleurs s'appliquent quasi exclusivement au syndicat disposant d'une convention collective ; autrement dit il s'agit d'un syndicat « établi ». Les salariés membres d'un syndicat « non établi » ne sont pas représentés par leur propre représentant en général. Il faut appréhender la situation privilégiée du syndicat à la lumière des faits suivants: en Suède, la plupart des sites de travail sont couverts par une convention collective, la densité syndicale est très élevée et la plupart des salariés d'un site sont membres du syndicat « établi ».

Le système suédois de participation des travailleurs repose sur un canal unique. La représentation se fait via l'entreprise; les salariés sont représentés par leurs syndicats uniquement. II n'existe pas d'instances de représentation parallèles, comme le comité d'entreprise. Le droit de participation des travailleurs est en fait un droit de codétermination pour les salariés exercé par le(s) syndicat(s).

En Norvège, la situation est différente. La loi norvégienne reconnaît les comités d'entreprise (bedriftsutvalg). Par ailleurs, la participation des travailleurs n'est pas, comme en Suède, concentrée dans les mains d'un syndicat «établi ». Les dispositions et l'ensemble des droits relatifs à la participation des travailleurs incluent tous les syndicats de manière égale.

Que ce soit en Norvège ou en Suède, l'information et la consultation sont soumises à des règles. En Norvège, ces règles ont été formulées dans les accords de base. Toutefois, puisqu'il ne suffit pas de mettre en œuvre les directives de l'UE à travers des conventions collectives, la directive sur l'information et la consultation des travailleurs (2002/14/CE) a été déployée en Norvège dans la loi sur l'environnement de travail de $2005^{40}$. Les dispositions

40 Voir la loi sur l'environnement de travail-de 2005 chapitre 8. 
de cette loi correspondent en grande partie au contenu des accords de base, mais ces derniers vont plus loin et combinent information/consultation unidirectionnelle avec une discussion bidirectionnelle visant à mettre en place des systèmes de négociation et de codétermination.

En Suède, les règles sur l'information et la consultation font, depuis les années 1970, partie de la législation et de la loi de codétermination de 1976. Le législateur part du principe que les partenaires sociaux agissent dans ce domaine et se mettent d'accord sur des règles de négociation et de codétermination.

La forme de représentation qui est probablement la plus importante dans la gestion quotidienne des entreprises se trouve dans les conventions collectives qui dotent les délégués syndicaux et les syndicats à l'échelle des entreprises de droits d'information, de consultation et de négociation sur de nombreuses questions. Les salariés sont représentés par leur représentant syndical. En Norvège, un représentant est élu directement par les membres du syndicat tandis qu'en Suède, il est nommé par le syndicat local.

En Suède, il n'existe pas de règles sur le nombre de représentants syndicaux qui doivent participer aux négociations avec l'employeur. C'est aux parties elles-mêmes de se mettre d'accord sur ce point. Par ailleurs, il n'existe pas de seuils de salariés pour que les règles soient applicables : la codétermination concerne les salariés de toutes les entreprises. En Norvège, la participation des travailleurs n'est applicable que dans les entreprises d'une certaine taille. Par exemple, le droit à l'information et à la consultation porte sur les sociétés ayant au moins cinquante salariés.

La réglementation norvégienne la plus importante en matière de codétermination porte sur «l'information, la coopération et la codétermination » dans les accords de base. Pour les entreprises sans syndicats, le chapitre de la loi sur l'environnement de travail de 2005 mettant en œuvre la directive sur l'information et la consultation s'applique. Par ailleurs, bien que ce point soit de moindre importance, un comité d'entreprise (bedriftsutvalg) doit être mis en place dans les entreprises de cent salariés au moins. La plupart des salariés ne bénéficient donc pas de comités d'entreprise.

Comme susmentionné, il n'existe pas de règles sur les comités d'entreprise en Suède. Au lieu de cela, la participation des travailleurs est exercée par les représentants syndicaux sur la base de la loi de codétermination de 1976 et des conventions collectives. Conformément à la loi, chaque syndicat, et pas seulement les syndicats " établis ", jouit d'un droit de négocier avec l'employeur sur les questions concernant les relations entre l'employeur et les membres du syndicat. Outre ce droit de négociation générale, les syndicats " établis » bénéficient d'un droit de négociation substantiel sur les sujets sur lesquels l'employeur possède un pouvoir de décision exclusif. Dans le cas de changements importants, l'employeur doit prendre l'initiative d'entrer en négociation avec le syndicat "établi» préalablement à la prise de décision. En Norvège, il existe des règles semblables dans les accords de base, sans toutefois que ne soit stipulé le droit de négocier pour le syndicat. La direction doit plutôt discuter du fonctionnement ordinaire de l'entreprise avec les délégués syndicaux. Si le problème concerne un seul salarié (licenciement, par exemple), l'employeur est tenu d'aborder la situation directement avec le salarié. Aucune obligation de ce type n'existe dans la législation suédoise.

Ces dispositions sur les négociations de codétermination sont importantes car le syndicat reçoit des informations à l'avance sur les changements potentiellement problématiques et car il a l'opportunité de poser des questions et de faire des commentaires 
et des suggestions à l'employeur. La décision finale revient toutefois entièrement à l'employeur.

En Suède, la loi de 1987 sur la représentation au conseil d'administration joue un rôle mineur pour la participation des travailleurs. La loi indique que, dans les entreprises de plus de vingt-cinq salariés, deux des membres du conseil d'administration doivent être nommés par les salariés, c'est-à-dire choisis par les syndicats signataire d'une convention collective. En Norvège, la représentation au conseil d'administration est régie par le droit des sociétés. Dans la loi de 1997 sur les sociétés ouvertes à responsabilité limitée et la loi de 1997 sur les sociétés privées à responsabilité limitée (lois sur les sociétés), il est précisé que les salariés des entreprises de trente salariés ou plus ont le droit d'élire des représentants des salariés au conseil d'administration.

\section{Conclusion}

Les travailleurs partagent avec les employeurs et les propriétaires de l'entreprise un même intérêt vis-à-vis de l'activité de l'entreprise. Cet intérêt va plus loin que celui des autres parties prenantes et est d'une autre nature ${ }^{41}$. En réalité, l'importance des intérêts des travailleurs pour l'activité ou l'entreprise serait réduite si les travailleurs étaient considérés comme n'importe quelle autre partie prenante, tels que les fournisseurs, les banques, les municipalités, les clients, etc. Dans une certaine mesure, les intérêts des parties prenantes limitent les pouvoirs des actionnaires, qui désignent sur la base de droits de propriété les décideurs au niveau de l'entreprise, au moins dans le secteur privé. Par conséquent, les travailleurs doivent être considérés, avec les actionnaires, comme des parties prenantes internes, ce qui contribue à légitimer leur influence aux côtés des actionnaires ${ }^{42}$.

Depuis le développement de la doctrine sur la flexibilisation à la fin des années 1970 et les derniers changements dans l'organisation du travail ${ }^{43}$, les droits des travailleurs se

41 Voir R. Fahlbeck, "What is prime : Work or Capital? », dans Ch Mestre, C. Sachs-Durand, et M. Storck, (réd.), Le travail humain au carrefour du droit et de la sociologie. Hommage au Professeur Nikitas Aliprantis, 2014, p. 389-396.

42 Cf. I. M. Hagen, et B.J. Mulder, «Why stakeholders?», dans S. Vitols, et J. Heuschmid, (éd.), European company law and the Sustainable Company : a stakeholder approach. Vol. II, 2013, 41-70, sp. 50-52. (Disponible au format numérique). Pour plus de détails, voir plutôt H. Sinzheimer, dans O. Kahn-Freund, et Th. Ramm, (éd.), Arbeitsrecht und Rechtssoziologie : gesammelte Aufsätze und Reden, 1976; R. Dukes, The Labour Constitution, 2014. cf. B.J. Mulder, Anställningen vid verksamhetsövergång, 2004, sp. p. 336-339, 354.

43 Cf. J. Prassl, M. Risak, Uber, «TaskRabbit \& Co : Platforms as Employers? Rethinking the Labour Law of Crowdwork», dans K. Ahlberg, et N. Bruun, (éd.), The New Foundations of Labour Law, 2017, p. 97-126. A. Westregård, "Collaborative economy - a new challenge for the social partners», dans K. Ahlberg, O. P. Herzfeld, J. Malmberg, N. Bruun i Sverige. En vänbok, 2017, p. 427-438. 
trouvent remis en cause, que ce soit au niveau national ou international ${ }^{44}$, à la fois par l'acteur traditionnel, autrement dit le capital ${ }^{45}$, et par les organisations internationales ${ }^{46}$ qui ont un impact sur les normes du travail et leur gestion ${ }^{47}$. Cela génère une défaillance de la démocratie étant donné que les décisions sont prises par des personnes autres que des représentants élus démocratiquement. Évidemment, le fait de contourner les processus démocratiques est en soi une remise en cause des droits des travailleurs. Dans cet environnement et face à cette évolution, il est important de préserver et de maintenir, mais aussi de développer les formes d'influence des travailleurs.

En Norvège et en Suède, le système de participation des travailleurs est basé sur la confiance. En Scandinavie, l'idée s'est avérée fructueuse. II est toutefois bien connu que l'encadrement juridique des droits collectifs dans un pays est difficilement transposable dans un autre pays ${ }^{48}$. et ce même s'il est possible de recourir à l'imitation et à l'adaptation et même si les pays scandinaves ont largement eu recours à ces techniques notamment pour élaborer la législation collective du travail en s'inspirant de l'Allemagne en particulier. Les structures fondamentales mises en place pour le droit collectif du travail présentent une stabilité et une importance considérables pour son évolution future. Ainsi, le cadre institutionnel et les systèmes demeurent tels qu'ils ont été établis à l'origine.

Au cours des décennies qui ont suivi la Seconde Guerre mondiale, les droits des employeurs de décider de manière unilatérale ont nettement reculé sur un certain nombre de sujets. Ils sont désormais contrôlés par des conventions collectives et des règlements statutaires. Les syndicats, notamment les syndicats " établis » (les syndicats signataires d'une convention collective), jouissent de droits majeurs leur permettant de conclure des accords essentiels pour le salarié individuellement. Les conventions collectives et les lois ne confèrent aux salariés aucun droit ne pouvant être géré par les syndicats avec l'employeur. En Suède, la loi de codétermination de 1976 peut servir d'exemple bien qu'elle n'ait pas d'équivalent dans la loi norvégienne. La loi n'offre aux salariés aucun droit relevant du domaine de la réglementation commune et ne régule pas la liberté de l'employeur de

44 Cf. A. lossa, "Collective Bargaining in a Globalised World : a Multi-dimensional Picture» dans L. Carlson, Ö. Edström, B. Nyström, (éd.), Globalisation, Fragmentation, Labour and Employment Law. A Swedish Perspective, 2016, p. 25-51.

45 Cf. R. Fahlbeck, "What is prime : Work or Capital?», dans Ch Mestre, C. Sachs-Durand, et M. Storck, (réd.), Le travail humain au carrefour du droit et de la sociologie. Hommage au Professeur Nikitas Aliprantis, 2014, p. 389-396.

46 Cf. M. Mwakagali, International Financial Institutions and Labour Standards, 2018. (Disponible au format numérique)

47 Voir également J. Fudge, «Blurring Legal Boundaries: Regulating for Decent Work», dans J. Fudge, S. McCrystal, et K. Sankaran, (éd.), Challenging the Legal Boundaries of Work Regulation, 2012, p. 1-26. (Disponible au format numérique)

48 Cf. O. Kahn-Freund, "On Uses and Misuses of Comparative Law», Modern Law Review, 1974, p. 1-27. 


\section{PARTICIPATION DES TRAVAILLEURS EN NORVÈGE gt EN SUÈDE}

diriger et d'organiser le travail avec des dispositions détaillées spécifiques. Au lieu de cela, la loi invite les syndicats « établis » à participer de leur propre chef au processus dynamique de direction et de d'organisation du travail. L'initiative unilatérale de l'employeur a été partiellement remplacée par une action bilatérale de l'employeur et des syndicats « établis ».

\section{BERNARD JOHANN MULDER}

Professeur de droit, Université d'Oslo, Norvège.

Thèmes de recherche : Droit du travail, droit international, droit européen, droit comparé et droit des contrats.

\section{Publications :}

A. Adlercreutz, B. J. Mulder, Avtal. Lärobok i allmän avtalsrätt (Contract. A textbook in general contract law), Wolters Kluwer 2017 (ISBN 9789139207924).

B. J. Mulder, Arbetstagarrepresentation i internationella koncernbolag. I: Niklas Bruun i Sverige, En vänbok (Worker representation in international group companies. In: Niklas Bruun in Sweden. Liber Amicorum). lustus förlag 2017, p. 275-288 (ISBN 9789177370109).

B. J. Mulder, The law concerning the election of employees' representatives in company bodies, Labour Law Journal 2017 p. 96-104 (ISSN 2031-9525). 